\title{
Quantum dephasing and decay of classical correlation functions in chaotic systems
}

\author{
Valentin V. Sokolov \\ Center for Nonlinear and Complex Systems, Università degli Studi dell'Insubria, Via Valleggio 11, 22100 Como, Italy \\ and Budker Institute of Nuclear Physics, Novosibirsk, Russia \\ Giuliano Benenti \\ Center for Nonlinear and Complex Systems, Università degli Studi dell'Insubria, Via Valleggio 11, 22100 Como, Italy \\ and CNISM and Istituto Nazionale di Fisica Nucleare, Sezione di Milano \\ Giulio Casati \\ Center for Nonlinear and Complex Systems, Università degli Studi dell'Insubria, Via Valleggio 11, 22100 Como, Italy \\ and CNISM and Istituto Nazionale di Fisica Nucleare, Sezione di Milano, Italy \\ and Department of Physics, National University of Singapore, Singapore 117542, Republic of Singapore \\ (Received 19 April 2005; revised manuscript received 11 October 2006; published 23 February 2007)
}

\begin{abstract}
We discuss the dephasing induced by internal classical chaotic motion in the absence of any external environment. To this end an extension of fidelity for mixed states is introduced, which we name allegiance. Such a quantity directly accounts for quantum interference and is measurable in a Ramsey interferometry experiment. We show that in the semiclassical limit the decay of the allegiance is exactly expressed, due to the dephasing, in terms of an appropriate classical correlation function. Our results are derived analytically for the case of a nonlinear driven oscillator and then numerically confirmed for the kicked rotor model.
\end{abstract}

DOI: 10.1103/PhysRevE.75.026213

PACS number(s): 05.45.Mt, 03.65.Sq, 05.45.Pq

\section{INTRODUCTION}

The study of the quantum manifestations of classical chaotic motion has greatly improved our understanding of quantum mechanics in relation to the properties of eigenvalues and eigenfunctions as well as to the time evolution of complex systems $[1,2]$. According to the Van Vleck-Gutzwiller semiclassical theory [3], quantum dynamics, even deeply in the semiclassical region, involves quantum interference of contributions from a large number of classical trajectories which exponentially grows with energy or, alternatively, with time. This interference manifests itself in various physical effects such as universal local spectral fluctuations, scars in the eigenstates, elastic enhancement in chaotic resonance scattering, weak localization in transport phenomena, and also in peculiarities of the wave packet dynamics and in the decay of the quantum Loschmidt echo (fidelity) [4]:

$$
F_{\psi}^{\circ}(t)=|\langle\dot{\psi}|\hat{f}(t)| \stackrel{\circ}{\psi}\rangle|^{2}=|\operatorname{Tr}[\hat{f}(t) \stackrel{\circ}{\rho}]|^{2}
$$

In Eq. (1), $\stackrel{\rho}{\rho}=|\stackrel{\circ}{\psi}\rangle\langle\stackrel{\sim}{\psi}|$ is the density matrix corresponding to the initial pure state $|\dot{\psi}\rangle \equiv|\psi(t=0)\rangle$. The unitary operators $\hat{U}_{0}(t)$ and $\hat{U}_{\varepsilon}(t)$ describe the unperturbed and perturbed evolutions of the system, according to the Hamiltonians $H_{0}$ and $H_{\varepsilon}=H_{0}+\varepsilon V$, respectively. Therefore, the echo operator $\hat{f}(t)$ $=\hat{U}_{0}^{\dagger}(t) \hat{U}_{\varepsilon}(t)$ represents the composition of a slightly perturbed Hamiltonian evolution with an unperturbed timereversed Hamiltonian evolution. The unperturbed part of the evolution can be perfectly excluded by making use of the interaction representation, thus obtaining

$$
\hat{f}(t)=T \exp \left[-i \frac{\varepsilon}{\hbar} \int_{0}^{t} d \tau \mathcal{H}(\tau)\right], \quad \mathcal{H}(\tau)=e^{(i / \hbar) H_{0} \tau} V e^{-(i / \hbar) H_{0} \tau}
$$

where $T$ is the time-ordering operator. Therefore, the fidelity (1) can be seen as the probability, for a system which evolves in accordance with the time-dependent Hamiltonian $\mathcal{H}(t)$ $=U_{0}^{\dagger}(t) V U_{0}(t)$, to stay in the initial state $|\dot{\psi}\rangle$ until time $t$.

The quantity (1), whose behavior depends on the interference of two wave packets evolving in a slightly different way, measures the stability of quantum motion under perturbations. Its decay has been investigated extensively in different parameter regimes and in relation to the nature of the corresponding classical motion (see [4-16] and references therein). Most remarkably, it turns out that a moderately weak coupling to a disordered environment, which destroys the quantum phase correlations, thus inducing decoherence, yields an exponential decay of fidelity, with a rate which is determined by the system's Lyapunov exponent and independent of the perturbation (coupling) strength [5]. In other words, quantum interference becomes irrelevant and the decay of fidelity is entirely determined by classical chaos. This result raises the interesting question whether the classical chaos, in the absence of any environment and only with a perfectly deterministic perturbation $V$, can by itself produce incoherent mixing of the quantum phases (dephasing) strongly enough to fully suppress the quantum interference. The answer is, generally, negative. Indeed, though the "effective" Hamiltonian evolves [see Eq. (2)] in accordance with the chaotic dynamics of the unperturbed system so that the actions along distant classical phase trajectories are statistically independent, still there always exist a lot of very close trajectories whose actions differ only by terms of the 
order of Planck's constant. Interference of such trajectories remains strong. We remark in this connection that any classical device is capable of preparing only incoherent mixed states described by diagonal density matrices. On the other hand, we should stress that a purely dynamical evolution, even if it starts from a diagonal initial mixed state, rapidly produces off-diagonal matrix elements which, generally, contain correlations thus manifesting quantum coherence. This must be opposed to the cases of stationary interaction with a disordered environment [5], thermal bath, or external white noise [14] when the correlations are washed out due to the external influence. So one could expect that, in the absence of any environment, quantum coherence is generated during dynamical evolution even if the initial state was fully incoherent. In this paper we show that, nevertheless, if the system is classically chaotic and the evolution starts from a wide and incoherent mixed state, then the initial incoherence persists due to the intrinsic classical chaos so that the quantum phases remain irrelevant.

Our paper is structured as follows. In Sec. II, we discuss two different possible measures, fidelity and allegiance, of the sensitivity of the evolution of a mixed quantum state to an external perturbation. In the limiting case of pure initial states, both fidelity and allegiance reduce to the well-known fidelity defined in Eq. (1). In this section, it is also pointed out that allegiance is the quantity naturally measured in Ramsey-type experiments. Section III introduces the kicked nonlinear oscillator which can serve as a model for an ion trap. A semiclassical computation of the decay of both fidelity and allegiance for this model in the chaotic regime is presented in Sec. IV (for initial pure coherent states) and Sec. $\mathrm{V}$ (for mixed states). This latter section analytically relates the decay of allegiance to that of a suitable correlation function of classical phases, thus establishing a link between quantum dephasing and decay of classical correlation functions in chaotic systems. This link is numerically confirmed in Sec. VI for the kicked rotor model, whose allegiance decay may be experimentally measured by means of cold atoms in an optical lattice. Finally, our conclusions are drawn in Sec. VII.

\section{MIXED STATES: FIDELITY VERSUS ALLEGIANCE}

In the case of a mixed initial state $\left(\stackrel{\circ}{\rho}=\Sigma_{k} p_{k}\left|\stackrel{\circ}{\psi}_{k}\right\rangle\left\langle\stackrel{\circ}{\psi}_{k}\right|\right.$, $\left.\Sigma_{k} p_{k}=1\right)$, fidelity is usually defined as [4]

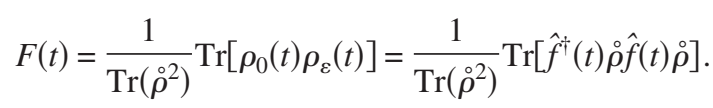

Note that for a pure state $\left(\rho^{2}=\rho, p_{k}=\delta_{k k}{ }^{\circ}\right)$ Eq. (3) reduces to (1).

Another interesting possibility is suggested by the experimental configuration with periodically kicked ion traps proposed in [17]. In such Ramsey-type interferometry experiments one directly accesses the fidelity amplitudes (see [17]) rather than their square moduli. Motivated by this consideration, we analyze in this paper the following natural generalization:

$$
\begin{aligned}
\mathcal{F}(t) & =|\operatorname{Tr}[\hat{f}(t) \stackrel{\circ}{\rho}]|^{2} \\
& =\left|\sum_{k} p_{k} f_{k}(t)\right|^{2} \\
& =\sum_{k} p_{k}^{2} F_{k}(t)+\sum_{k, k^{\prime}}\left(1-\delta_{k k^{\prime}}\right) p_{k} p_{k^{\prime}} f_{k}(t) f_{k^{\prime}}^{*}(t),
\end{aligned}
$$

which is obtained by directly extending the formula (1) to the case of an arbitrary mixed initial states $\stackrel{\circ}{\rho}$. Below we refer to this new quantity as allegiance. The first term on the righthand side (RHS) is the sum of fidelities $F_{k}=\left|f_{k}\right|^{2}$ $=\left|\left\langle\dot{\psi}_{k}|\hat{f}| \dot{\psi}_{k}\right\rangle\right|^{2}$ of the individual pure initial states with weights $p_{k}^{2}$, while the second, interference term, depends on the relative phases of fidelity amplitudes. If the number $K$ of pure states $\left|\dot{\psi}_{k}\right\rangle$ which form the initial mixed state is large, $K \gg 1$, so that $p_{k} \sim 1 / K$ for $k \leqslant K$ and zero otherwise, the first term is $\sim 1 / K$ at the initial moment $t=0$ while the second term $\sim 1$. Therefore, in the case of a wide mixture, the decay of the function $\mathcal{F}(t)$ is determined by the interference terms in Eq. (4).

The allegiance $\mathcal{F}(t)$ [Eq. (4)] is different from the mixedstate fidelity $F(t)$ [Eq. (3)] as well as from the incoherent sum

$$
\overline{F(t)}=\sum_{k} p_{k}\left|f_{k}\right|^{2}
$$

of pure-state fidelities typically considered in the literature. This latter quantity corresponds to averaging pure-state fidelities, instead of fidelity amplitudes as in allegiance. Quantities (3) and (5) both contain only transition probabilities induced by the echo operator, $W_{k k^{\prime}}=\left|\left\langle\dot{\psi}_{k}|\hat{f}(t)| \dot{\circ}_{k^{\prime}}\right\rangle\right|^{2}$. Indeed, we can write the mixed-state fidelity (3) as $F(t)$ $=\frac{1}{\operatorname{Tr}\left(\dot{\rho}^{2}\right)} \sum_{k, k^{\prime}} p_{k} p_{k^{\prime}} W_{k k^{\prime}}$. In contrast, the allegiance $\mathcal{F}$ directly accounts for the quantum interference and can be expected to retain quantal features even in the deep semiclassical region.

Notice that the quantity $\mathcal{F}(t)$ is naturally measured in experiments performed on cold atoms in optical lattices [18] and in atom optics billiards [19] and proposed for superconducting nanocircuits [20]. This quantity is reconstructed after averaging the amplitudes over several experimental runs (or many atoms). Each run may differ from the previous one in the external noise realization and/or in the initial conditions drawn, for instance, from a thermal distribution [19]. Note that the averaged (over noise) fidelity amplitude can exhibit rather different behavior with respect to the averaged fidelity [20].

\section{ION TRAPS: THE DRIVEN NONLINEAR OSCILLATOR MODEL}

As a model for a single ion trapped in an anharmonic potential we consider a quartic oscillator driven by a linear multimode periodic force $g(t)$, 


$$
H_{0}=\hbar \omega_{0} n+\hbar^{2} n^{2}-\sqrt{\hbar}\left(a+a^{\dagger}\right) g(t), \quad n=a^{\dagger} a,\left[a, a^{\dagger}\right]=1 .
$$

In our units, the time and parameters $\hbar, \omega_{0}$ as well as the strength of the driving force are dimensionless. The period of the driving force is set to 1 . We use below the basis of coherent states $|\alpha\rangle$ which minimize in the semiclassical domain the action-angle uncertainty relation and which therefore constitutes the most adequate reference frame in the semiclassical domain $[21,22]$. These states are fixed by the eigenvalue problem $a|\alpha\rangle=\frac{\alpha}{\sqrt{\hbar}}|\alpha\rangle$, where $\alpha$ is a complex number which does not depend on $\hbar$. The corresponding normalized phase density (Wigner function) is $\rho_{\alpha}\left(\alpha^{*}, \alpha\right)=\frac{2}{\pi \hbar} e^{-2} \frac{|\alpha-\alpha|^{2}}{\hbar}$ and occupies a cell of volume $\sim \hbar$ in the phase plane $\left(\alpha^{*}, \alpha\right)$. Coherent interfering contributions in Van Vleck-Gutzwiller semiclassical theory just come from the "shadowing" trajectories which originate from such phase regions. In the classical limit $(\hbar \rightarrow 0)$ the above phase density reduces to Dirac's $\delta$ function, thus fixing a unique classical trajectory.

Since the scalar product of two coherent states equals $\left\langle\alpha^{\prime} \mid \alpha\right\rangle=\exp \left(-\frac{\left|\alpha^{\prime}-\alpha\right|^{2}}{2 \hbar}+\frac{i}{\hbar} \operatorname{Im}\left(\alpha^{\prime *} \alpha\right)\right)$, they become orthogonal in the classical limit $\hbar \rightarrow 0$. The Hamiltonian matrix $\left\langle\alpha^{\prime}\left|H_{0}\right| \alpha\right\rangle$ is diagonal in this limit and reduces to the classical Hamiltonian function $H_{0}^{(c)}=\omega_{0}\left|\alpha_{c}\right|^{2}+\left|\alpha_{c}\right|^{4}-\left(\alpha_{c}^{*}+\alpha_{c}\right) g(t)$. The complex variables $\alpha_{c}$ and $i \alpha_{c}{ }^{*}$ are canonically conjugated and are related to the classical action-angle variables $I_{c}$ and $\theta_{c}$ via $\alpha_{c}=\sqrt{I_{c}} e^{-i \theta_{c}}$ and $\alpha_{c}^{*}=\sqrt{I_{c}} e^{i \theta_{c}}$. The action satisfies the nonlinear integral equation

$$
I_{c}(t)=\left|\dot{\alpha}_{c}+i \int_{0}^{t} d \tau g(\tau) e^{i \varphi_{c}(\tau)}\right|^{2} \equiv\left|a_{c}(t)\right|^{2},
$$

where $\alpha_{c}(t)=a_{c}(t) e^{-i \varphi_{c}(t)}$ and $\varphi_{c}(t)=\int_{0}^{t} d \tau\left[\omega_{0}+2 I_{c}(\tau)\right]$.

When the strength of the driving force exceeds some critical value, the classical motion becomes chaotic and the phase $\varphi_{c}(t)$ becomes random so that its autocorrelation function decays exponentially with time:

$$
\left|\int d^{2} \stackrel{\circ}{\alpha} P_{\alpha_{c}}(\stackrel{\circ}{\alpha}, \stackrel{\circ}{\alpha}) e^{i\left[\varphi_{c}(t)-\varphi_{c}(0)\right]}\right|^{2}=\exp \left(-t / \tau_{c}\right),
$$

where $\mathcal{P}_{\alpha_{c}}\left(\stackrel{\circ}{\alpha}^{*}, \stackrel{\circ}{\alpha}\right)$ is the initial density distribution in the phase plane. Moreover, Eqs. (7) and (8) yield the diffusive growth $\left\langle I_{c}(t)\right\rangle=\stackrel{\circ}{I}_{c}+D t$ of the mean action, where $\stackrel{\circ}{I}_{c}=\left\langle I_{c}(t=0)\right\rangle$. By numerical integration of the classical equations of motion we have verified this statement as well as the exponential decay (8).

\section{SEMICLASSICAL EVOLUTION AND FIDELITY DECAY FOR COHERENT STATES}

In what follows, we analytically evaluate both the fidelity $F_{\alpha}(t)$ for a pure coherent quantum state $|\stackrel{\circ}{\alpha}\rangle$ (this section) and the allegiance $\mathcal{F}(t)$ for an incoherent mixed state (Sec. V) by treating the unperturbed motion semiclassically. Our semiclassical approach allows us to compute these quantities even for quantally strong perturbations $\sigma=\varepsilon / \hbar \gtrsim 1$.
The semiclassical evolution $\left|\psi_{\dot{\alpha}}(t)\right\rangle=\hat{U}_{0}(t)|\stackrel{\circ}{\alpha}\rangle$ of an initial coherent state when the classical motion is chaotic has been investigated in [23]. With the help of Fourier transformation one can linearize the chronological exponent $\hat{U}_{0}(t)$ with respect to the operator $n$, thus arriving at the following Feynman's path-integral representation in the phase space:

$$
\begin{aligned}
\left|\psi_{\alpha}(t)\right\rangle= & \int \prod_{\tau} \frac{d \lambda(\tau)}{\sqrt{4 \pi i \hbar}} \exp \left\{\frac{i}{4 \hbar} \int_{0}^{t} d \tau \lambda^{2}(\tau)-\frac{i}{\hbar} \operatorname{Im}\left[\beta_{\lambda}(t)\right]\right\} \\
& \times\left|\alpha_{\lambda}(t)\right\rangle .
\end{aligned}
$$

The functions with the subscript $\lambda$ are obtained by substituting $2 I_{c} \Rightarrow \lambda$ in the corresponding classical functions: $\alpha_{\lambda}(t)$ $=\left[\stackrel{\circ}{\alpha}+i \int_{0}^{t} d \tau g(\tau) e^{i \varphi_{\lambda}(\tau)}\right] e^{-i \varphi_{\lambda}(t)}$ and $\beta_{\lambda}(t)=-i \int_{0}^{t} d \tau g(\tau) \alpha_{\lambda}(\tau)$, where $\varphi_{\lambda}(t)=\int_{0}^{t} d \tau\left[\omega_{0}+\lambda(\tau)\right]$.

As an example we choose a perturbation $V$ which corresponds to a small, time-independent variation of the linear frequency: $\omega_{0} \rightarrow \omega_{0}+\varepsilon$ [24]. For convenience, we consider the symmetric fidelity operator $\hat{f}(t)=\hat{U}_{(+)}^{\dagger}(t) \hat{U}_{(-)}(t)$, where the evolution operators $\hat{U}_{( \pm)}(t)$ correspond to the Hamiltonians $H_{( \pm)}=H_{0} \pm \frac{1}{2} \varepsilon n$, respectively. Using Eq. (9) we express $f_{\dot{\alpha}}(t)$ as a double path integral over $\lambda_{1}$ and $\lambda_{2}$. A linear change of variables $\lambda_{1}(\tau)=2 \mu(\tau)-\frac{1}{2} \hbar \nu(\tau)$ and $\lambda_{2}(\tau)=2 \mu(\tau)+\frac{1}{2} \hbar \nu(\tau)$ entirely eliminates the Planck's constant from the integration measure. After making the shift $\nu(t) \rightarrow \nu(t)-\varepsilon / \hbar$ we obtain

$$
\begin{aligned}
\mathrm{f}_{\alpha}(t)= & \int \prod_{\tau} \frac{d \mu(\tau) d \nu(\tau)}{2 \pi} \exp \left\{i \sigma \int_{0}^{t} d \tau \mu(\tau)\right. \\
& -i \int_{0}^{t} d \tau \mu(\tau) \nu(\tau)+\frac{i}{\hbar} J[\mu(\tau), \nu(\tau)] \\
& \left.-\frac{1}{2 \hbar} R[\mu(\tau), \nu(\tau)]\right\},
\end{aligned}
$$

where the functionals $\mathcal{J}$ and $\mathcal{R}$ equal

$$
\begin{gathered}
\mathcal{J}=\hbar \int_{0}^{t} d \tau \nu(\tau)\left|a_{\mu}(\tau)\right|^{2}+O\left(\hbar^{3}\right), \\
\mathcal{R}=\hbar^{2}\left|\int_{0}^{t} d \tau \nu(\tau) a_{\mu}(\tau)\right|^{2}+O\left(\hbar^{4}\right),
\end{gathered}
$$

and vanish in the limit $\hbar=0$. The quantities with the subscript $\mu$ are obtained by setting $\nu(\tau) \equiv 0$ (in particular, $a_{\mu}(t)$ $=\alpha_{\mu}(t) e^{i \varphi_{\mu}(t)}$, with $\alpha_{\mu}(t)=\left[\stackrel{\circ}{\alpha}+i \int_{0}^{t} d \tau g(\tau) e^{i \varphi_{\mu}(\tau)}\right] e^{-i \varphi_{\mu}(t)}$ and $\left.\varphi_{\mu}(t)=\int_{0}^{t} d \tau\left[\omega_{0}+2 \mu(t)\right]\right)$. In the lowest ("classical") approximation when only the term $\sim \hbar$ from Eqs. (10) is kept, the $\nu$ integration results in the $\delta$ function $\Pi_{\tau} \delta\left[\mu(\tau)-\left|a_{\mu}(\tau)\right|^{2}\right]$, so that $\mu(t)$ coincides with the classical action $I_{c}(t)$ [see Eq. (7)]. The only contribution comes then from the periodic classical orbit which passes through the phase point $\stackrel{\circ}{\alpha}$. The corresponding fidelity amplitude is simply equal to $f_{\dot{\alpha}}(t)=\exp \left[i \sigma \int_{0}^{t} d \tau I_{c}(\tau)\right]$.

The first correction, given by the term $\sim \hbar^{2}$ in the functional $\mathcal{R}$, describes the quantum fluctuations. The functional integration still can be carried out exactly [23]. Now a bunch 
of trajectories contributes, which satisfy the equation $\mu(t ; \delta)=\left|\delta+a_{\mu}(t)\right|^{2}-|\delta|^{2}$ for all $\delta$ within a quantum cell $\sim \hbar$. This equation can still be written in the form of the classical equation (7) if we define the classical action along a given trajectory as $\widetilde{I}_{c}(t)=\left|a_{\mu}(t)+\delta\right|^{2}=\mu(t ; \delta)+|\delta|^{2}$ $=I_{c}\left(\omega_{0}-2|\delta|^{2} ; \stackrel{\circ}{\alpha}^{*}+\delta^{*}, \stackrel{\circ}{\alpha}+\delta ; t\right)$. For any given $\delta$ this equation describes the classical action of a nonlinear oscillator with linear frequency $\omega_{0}-2|\delta|^{2}$, which evolves along a classical trajectory starting from the point $\stackrel{\circ}{\alpha}+\delta$. One then obtains (up to the irrelevant overall phase factor $e^{-i \omega_{0} t / 2 \hbar}$ )

$$
f_{\dot{\alpha}}(t)=\frac{2}{\pi \hbar} \int d^{2} \delta e^{-(2 / \hbar)|\delta|^{2}} \exp \left\{i \frac{\sigma}{2}\left[\widetilde{\varphi}_{c}(t)-\widetilde{\varphi}_{c}(0)\right]\right\},
$$

where the "classical" phase $\widetilde{\varphi}_{c}(t)=\varphi_{c}\left(\omega_{0}-2|\delta|^{2} ; \stackrel{\circ}{\alpha}+\delta^{*}, \stackrel{\circ}{\alpha}\right.$ $+\delta ; t)=\int_{0}^{t} d \tau\left[\omega_{0}-2|\delta|^{2}+2 \widetilde{I}_{c}(\tau)\right]$. This expression gives the fidelity amplitude in the "initial value representation" $[11,25]$. We stress that the fidelity $F_{\alpha}=\left|f_{\alpha}\right|^{2}$ does not decay in time if the quantum fluctuations described by the integral over $\delta$ in Eq. (11) are neglected.

On the initial stage of the evolution, while the phases $\tilde{\varphi}_{c}(t)$ are not yet randomized and still remember the initial conditions, we can expand $\widetilde{\varphi}_{c}(t)$ over the small shifts $\delta$. Keeping only linear and quadratic terms in Eq. (11) we get after double Gaussian integration

$$
\begin{aligned}
F_{\dot{\alpha}}(t)= & {\left[1+\left(\frac{\varepsilon}{2}\right)^{2}\left(\frac{\partial \varphi_{c}(t)}{\partial \omega_{0}}\right)^{2}\right]^{-1} } \\
& \times \exp \left\{-\frac{\varepsilon^{2}}{4 \hbar}\left|\frac{\partial \varphi_{c}(t)}{\partial \dot{\alpha}}\right|^{2}\left[1+\left(\frac{\varepsilon}{2}\right)^{2}\left(\frac{\partial \varphi_{c}(t)}{\partial \omega_{0}}\right)^{2}\right]^{-1}\right\} .
\end{aligned}
$$

Due to exponential local instability of the classical dynamics, the derivatives $\left|\frac{\partial \varphi_{c}(t)}{\partial \dot{\alpha}}\right|,\left|\frac{\partial \varphi_{c}(t)}{\partial \omega_{0}}\right| \propto e^{\Lambda t}$ where $\Lambda$ is the Lyapunov exponent. So the function (12), up to time $t \ll \frac{1}{\Lambda} \ln \frac{2}{\varepsilon}$, decays superexponentially: $\quad F_{\dot{\alpha}}(t) \approx \exp \left(-\frac{\varepsilon^{2}}{4 \hbar}\left|\frac{\partial \varphi_{c}(t)}{\partial \dot{\alpha}}\right|^{2}\right)=\exp \left(-\frac{\varepsilon^{2}}{4 \hbar} e^{2 \Lambda t}\right)$ $[10,14]$. During this time the contribution of the averaging over the initial Gaussian distribution in the classical $\stackrel{\circ}{\alpha}$ phase plane dominates while the influence of the quantum fluctuations of the linear frequency described by the $\omega_{0}$ derivative remains negligible. Such a decay has, basically, a classical nature [15], and Planck's constant appears only as the size of the initial distribution. On the contrary for larger times the quantum fluctuations of the frequency control the fidelity decay, which becomes exponential: $F_{\alpha}(t) \propto\left(\frac{\partial \varphi_{c}(t)}{\partial \omega_{0}}\right)^{-2}$ $=\exp (-2 \Lambda t)$.

\section{MIXED STATES: ALLEGIANCE VERSUS CLASSICAL CORRELATION FUNCTIONS}

Now we discuss the decay of the allegiance $\mathcal{F}(t)$ when the initial condition corresponds to a broad incoherent mixture. More precisely, we consider a mixed initial state represented by a Glauber's diagonal expansion [26] $=\int d^{2} \stackrel{\circ}{\alpha} \mathcal{P}\left(|\stackrel{\circ}{\alpha}-\stackrel{\circ}{\alpha}|^{2}\right)|\stackrel{\circ}{\alpha}\rangle\langle\stackrel{\circ}{\alpha}|$ with a wide positive-definite weight function $\mathcal{P}$ which covers a large number of quantum cells. Note that here and in the following we assume that the initial mixture is isotropically distributed in the phase plane around a fixed point $\stackrel{\circ}{\alpha}_{c}$, with density $\mathcal{P}_{\alpha_{\alpha}}(\stackrel{\circ}{\alpha}, \stackrel{\circ}{\alpha})=\mathcal{P}\left(\left|\stackrel{\circ}{\alpha}-\stackrel{\circ}{\alpha}_{c}\right|^{2}\right)$. Then allegiance (4) equals $\mathcal{F}\left(t ; \stackrel{\circ}{\alpha}_{c}\right)=\left|f\left(t ; \stackrel{\circ}{\alpha}_{c}\right)\right|^{2}$, where

$$
\begin{aligned}
f\left(t ; \stackrel{\circ}{\alpha}_{c}\right) \equiv & \int d^{2} \stackrel{\circ}{\alpha} \mathcal{P}\left(\left|\stackrel{\circ}{\alpha}-\stackrel{\circ}{\alpha}_{c}\right|^{2}\right) f_{\dot{\alpha}}(t) \\
\approx & \frac{2}{\pi \hbar} \int d^{2} \delta e^{-(2 / \hbar)|\delta|^{2}} \\
& \times \int d^{2} \stackrel{\circ}{\alpha} P\left(\left|\stackrel{\circ}{\alpha}-\left(\stackrel{\circ}{\alpha}_{c}+\delta\right)\right|^{2}\right) e^{i(\sigma / 2)\left[\bar{\varphi}_{c}(t)-\bar{\varphi}_{c}(0)\right]},
\end{aligned}
$$

with $\bar{\varphi}_{c}(t)=\varphi_{c}\left(\omega_{0}-2|\delta|^{2} ; \stackrel{\circ}{\alpha}^{*}, \stackrel{\circ}{\alpha} ; t\right)$. The inner integral over $\stackrel{\circ}{\alpha}$ looks like a classical correlation function. In the regime of classically chaotic motion this correlator cannot appreciably depend on either the exact location of the initial distribution in the classical phase space or on small variations of the value of the linear frequency. Indeed, though an individual classical trajectory is exponentially sensitive to variations of initial conditions and system parameters, the manifold of all trajectories which contribute to (13) is stable [7]. Therefore, we can fully disregard the $\delta$ dependence of the integrand, thus obtaining

$$
f\left(t ; \stackrel{\circ}{\alpha}_{c}\right) \approx \int d^{2} \stackrel{\circ}{\alpha} P\left(\left|\stackrel{\circ}{\alpha}-\stackrel{\circ}{\alpha}_{c}\right|^{2}\right) \exp \left\{i \frac{\sigma}{2}\left[\varphi_{c}(t)-\varphi_{c}(0)\right]\right\} \text {. }
$$

This is the main result of our paper which directly relates the decay of a quantum quantity, the allegiance, to that of correlation functions of classical phases [see Eq. (8)]. No quantum feature is present in the RHS of (14).

The decay pattern of the function $\mathcal{F}(t)=\left|f\left(t ; \stackrel{\circ}{\alpha}_{c}\right)\right|^{2}$ depends on the value of the parameter $\sigma=\varepsilon / \hbar$. In particular, for $\sigma \ll 1$, we recover the well known Fermi golden rule (FGR) regime. Indeed, in this case the cumulant expansion can be used, $\ln f\left(t ; \stackrel{\circ}{\alpha}_{c}\right)=\sum_{\kappa=1}^{\infty} \frac{(i \sigma)^{\kappa}}{\kappa !} \chi_{\kappa}(t)$. All the cumulants are real; hence, only the even ones are significant; The lowest of them,

$$
\begin{aligned}
\chi_{2}(t) & =\int_{0}^{t} d \tau_{1} \int_{0}^{t} d \tau_{2}\left\langle\left[I_{c}\left(\tau_{1}\right)-\left\langle I_{c}\left(\tau_{1}\right)\right\rangle\right]\left[I_{c}\left(\tau_{2}\right)-\left\langle I_{c}\left(\tau_{2}\right)\right\rangle\right]\right\rangle \\
& \equiv \int_{0}^{t} d \tau_{1} \int_{0}^{t} d \tau_{2} K_{I}\left(\tau_{1}, \tau_{2}\right),
\end{aligned}
$$

is positive. Assuming that the classical autocorrelation function decays exponentially, $K_{I}\left(\tau_{1}, \tau_{2}\right)=\left\langle\left(\Delta I_{c}\right)^{2}\right\rangle$ $\times \exp \left(-\left|\tau_{1}-\tau_{2}\right| / \tau_{I}\right)$ with some characteristic time $\tau_{I}$, we obtain $\chi_{2}(t)=2\left\langle\left(\Delta I_{c}\right)^{2}\right\rangle \tau_{I} t=2 K t$ for the times $t>\tau_{I}$ and arrive, finally, at the FGR decay law $\mathcal{F}\left(t ; \stackrel{\circ}{\alpha}_{c}\right)=\exp \left(-2 \sigma^{2} K t\right)[6-8]$. Here $K=\int_{0}^{\infty} d \tau K_{I}(\tau, 0)=\left\langle\left(\Delta I_{c}\right)^{2}\right\rangle \tau_{I}$.

The significance of the higher connected correlators $\chi_{\kappa \geqslant 4}(t)$ grows with the increase of the parameter $\sigma$. When this parameter roughly exceeds 1 , the cumulant expansion 
fails and the FGR approximation is no longer valid. In the regime $\sigma \gtrsim 1$, the decay rate of the function $\mathcal{F}\left(t ; \stackrel{\circ}{\alpha}_{c}\right)$ $=\left|f\left(t ; \stackrel{\circ}{\alpha}_{c}\right)\right|^{2}$ ceases to depend on $\sigma[27]$ and coincides with the decay rate $1 / \tau_{c}$ of the correlation function (8),

$$
\mathcal{F}\left(t ; \stackrel{\circ}{\alpha}_{c}\right)=\exp \left(-t / \tau_{c}\right) .
$$

This rate is intimately related to the local instability of the chaotic classical motion though it is not necessarily given by the Lyapunov exponent $\Lambda$ (it is worth noting in this connection that the Lyapunov exponent diverges in our driven nonlinear oscillator model).

Returning to the averaged fidelity (5), it can be decomposed into the sum of a mean $\left(|\bar{f}|^{2} \equiv \mathcal{F}\right)$ and a fluctuating part:

$$
\overline{F(t)}=\mathcal{F}(t)+\overline{|f(t)-\overline{f(t)}|^{2}} .
$$

As we have already stressed above, the allegiance $\mathcal{F}$ and the average fidelity $\bar{F}$ are quite different in nature. Nevertheless, due to the dephasing induced by classical chaos, the decays of these two quantities are tightly connected: they both decay with the same rate though the decay of $\overline{F(t)}$ is delayed by a time $t_{d}$. To show this, let us make use of the Fourier transform of the fidelity operator:

$$
\hat{f}(t)=\frac{1}{\pi} \int d^{2} \eta q\left(\eta^{*}, \eta ; t\right) \hat{D}(\eta), \quad q\left(\eta^{*}, \eta ; t\right)=\operatorname{Tr}\left[\hat{D}^{\dagger}(\eta) \hat{f}(t)\right]
$$

where $\hat{D}(\eta)=\exp \left(\eta a^{\dagger}-\eta^{*} a\right)$ is the displacement operator of coherent states. The Fourier transform $q$ satisfies the obvious initial condition $q\left(\eta^{*}, \eta ; 0\right)=\pi \delta^{(2)}(\eta)$. On the other hand, unitarity of the fidelity operator yields

$$
\frac{1}{\pi} \int d^{2} \kappa e^{\left(\omega \kappa^{*}-\omega^{*} \kappa\right) / 2} Q(\omega, \kappa ; t)=\pi \delta^{(2)}(\omega) .
$$

Here the shorthand

$$
Q(\omega, \kappa ; t) \equiv q^{*}\left(\kappa^{*}-\frac{1}{2} \omega^{*}, \kappa-\frac{1}{2} \omega ; t\right) q\left(\kappa^{*}+\frac{1}{2} \omega^{*}, \kappa+\frac{1}{2} \omega ; t\right)
$$

has been used. The function $Q$ factorizes at the initial moment $t=0$ as $Q(\omega, \kappa ; 0)=\pi^{2} \delta^{(2)}(\kappa) \delta^{(2)}(\omega)$.

From Eqs. (4), (5), and (18) we obtain

$$
\mathcal{F}(t)=\frac{1}{\pi^{2}} \int d^{2} \omega e^{-(\Delta / 2 \hbar+1 / 4)|\omega|^{2}} \int d^{2} \kappa e^{-(2 \Delta / \hbar+1)|\kappa|^{2}} Q(\omega, \kappa ; t)
$$

and

$$
\overline{F(t)}=\frac{1}{\pi^{2}} \int d^{2} \omega e^{-(\Delta / \hbar+1 / 4)|\omega|^{2}} \int d^{2} \kappa e^{-|\kappa|^{2}} Q(\omega, \kappa ; t) .
$$

Since we have assumed that the width of the initial mixture $\Delta \gg \hbar$, the essential difference between the two latter expressions lies in the $\kappa$-integration domain which is determined by the exponential factor. However, at the initial moment $t=0$ this difference is not relevant since the function $Q$ is sharply peaked. Then, in the evolution process the function $Q$ widens so that the exponential factors begin to define the integration range. This effect, in Eq. (21), takes place almost from the very beginning and therefore, after a very short time,

$$
\mathcal{F}(t) \approx\left(\frac{\hbar}{\Delta}\right)^{2}|q(0,0 ; t)|^{2} \approx \exp \left(-t / \tau_{c}\right) .
$$

[In the second equality we took into account the previously obtained result (16).] On the contrary, the cut in the integration over $\kappa$ is appreciably weaker in Eq. (22). As long as the factor $Q$ still decays faster than $e^{-|\kappa|^{2}}$ the latter can be substituted by unity and the $\kappa$ integration gives approximately $\pi \delta^{(2)}(\omega)$ as in the unitarity condition (19). Up to this time $t_{d}$ the function $\overline{F(t)}$ remains very close to 1 . When $t>t_{d}$,

$$
\overline{F\left(t>t_{d}\right)} \approx \frac{\hbar}{\Delta}\left|q\left(0,0 ; t>t_{d}\right)\right|^{2} \approx \exp \left(-\frac{t-t_{d}}{\tau_{c}}\right) .
$$

Comparison of the two last equations allows us to estimate the delay time to be $t_{d}=\tau_{c} \ln \frac{\Delta}{\hbar}$. On the other hand, for the Peres' mixed-state fidelity (3) we get

$$
F(t)=\frac{1}{\pi^{2}} \int d^{2} \omega e^{-(\Delta / 2 \hbar+1 / 4)|\omega|^{2}} \int d^{2} \kappa e^{-(\hbar / 2 \Delta)|\kappa|^{2}} Q(\omega, \kappa ; t) .
$$

When $\Delta \gg \hbar$ the $\kappa$ integration is not cut and the decay of $F(t)$, contrary to Eqs. (23) and (24), is determined by the large- $\kappa$ asymptotic behavior of the function $q$. The fidelity (3) and (25) has a well-defined classical limit which coincides with the classical fidelity $[8,15,16]$ and decays due to the phase flow out of the phase volume initially occupied. This has nothing to do with dephasing. In particular, if the initial distribution is uniform in the whole phase space, the fidelity (3) never decays.

In closing this section, we discuss possible choices of the initial mixture $\mathcal{P}$. If we choose $\stackrel{\circ}{\alpha}_{c}=0$, the initial mixed state reads as follows in the eigenbasis of the Hamiltonian $H^{(0)}=\hbar \omega_{0} n+\hbar^{2} n^{2}$ of the autonomous oscillations:

$$
\stackrel{\circ}{\rho}=\int d^{2} \stackrel{\circ}{\alpha} \mathcal{P}\left(|\stackrel{\alpha}{\alpha}|^{2}\right)|\stackrel{\circ}{\alpha}\rangle\left\langle\stackrel{\circ}{\alpha}\left|=\sum_{n=0}^{\infty} \stackrel{\circ}{\rho}_{n}\right| n\right\rangle\langle n|,
$$

where

$$
\stackrel{\circ}{\rho}_{n}=\frac{\pi}{n !} \int_{0}^{\infty} d \stackrel{\circ}{ } \mathcal{P}(\stackrel{\circ}{I}) e^{-\stackrel{\circ}{I} \hbar}(\stackrel{\circ}{I} / \hbar)^{n}, \quad \stackrel{\circ}{I}=|\stackrel{\circ}{\alpha}|^{2}
$$

This formula is inverted as

$$
\mathcal{P}\left(\stackrel{\circ}{I)}=\frac{e^{I / \hbar}}{2 \pi^{2}} \hbar \int_{-\infty}^{\infty} d k e^{i k I} R(k), \quad R(k)=\sum_{n=0}^{\infty} \stackrel{\circ}{\rho}_{n}(-i \hbar k)^{n} .\right.
$$

Therefore, our initial state is a totally incoherent mixture of the eigenstates $|n\rangle$. In particular, it can be the thermal distribution $\stackrel{\circ}{\rho}_{n}=\exp \left(-E^{(0)} / T\right)$, a choice of particular interest for experimental investigations. 


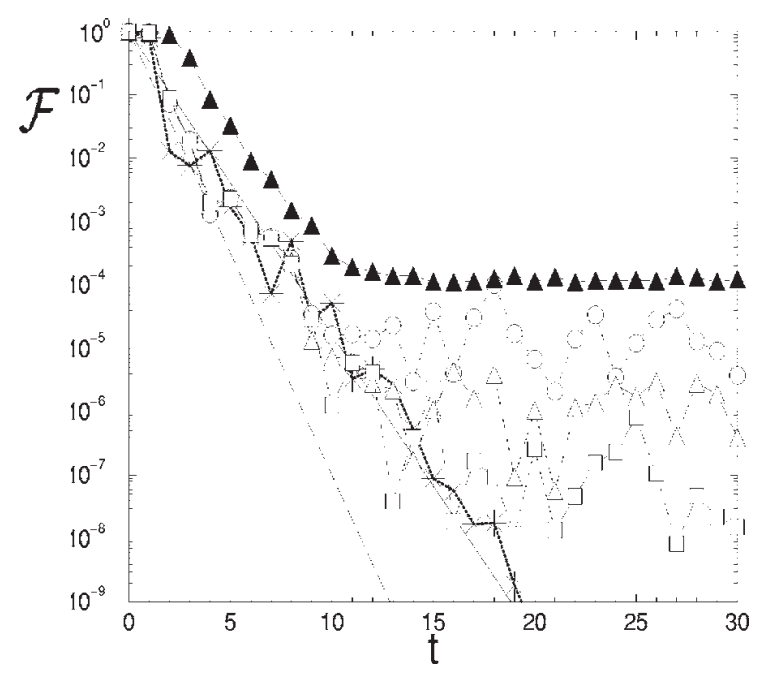

FIG. 1. Decay of the allegiance $\mathcal{F}$ for the kicked rotor model with $K=10$, perturbation strength $\epsilon / \hbar=1.1, \hbar=3.1 \times 10^{-3}$ (circles), $7.7 \times 10^{-4}$ (open triangles), and $1.9 \times 10^{-4}$ (squares). Solid triangles show the average fidelity $\bar{F}$ for $\hbar=7.7 \times 10^{-4}$. Asterisks give the decay of the classical angular correlation function. The straight lines denote exponential decay with rates given by the Lyapunov exponent $\Lambda \approx \ln (K / 2)=1.61$ (dashed line) and by the exponent $\Lambda_{1} \equiv \tau_{c}^{-1}=1.1[10]$ (solid line)

\section{FIDELITY DECAY IN OPTICAL LATTICES: THE KICKED ROTOR MODEL}

As a second example, we consider the kicked rotor model [28], described by the Hamiltonian $H=\frac{p^{2}}{2}$ $+K \cos \theta \Sigma_{m} \delta(t-m)$, with $[p, \theta]=-i \hbar$. The kicked rotor has been experimentally implemented by cold atoms in a standing wave of light [29-32]. Moreover, the fidelity amplitude for this model can be measured if one exploits atom interferometry $[17,18,33,34]$. The classical limit corresponds to the effective Planck constant $\hbar \rightarrow 0$. We consider this model on the torus, $0 \leqslant \theta<2 \pi,-\pi \leqslant p<\pi$. The allegiance $\mathcal{F}$ is computed for a static perturbation $\epsilon p^{2} / 2$, the initial state being a mixture of Gaussian wave packets uniformly distributed in the region $0.2 \leqslant \theta / 2 \pi \leqslant 0.3,0.3 \leqslant p / 2 \pi \leqslant 0.4$. In Fig. 1 we show the decay of $\mathcal{F}(t)$ in the semiclassical regime $\hbar \ll 1$ and for a quantally strong perturbation $\epsilon / \hbar \sim 1$. It is clearly seen that the allegiance follows the decay of the classical angular correlation function $\left.|\exp \{i \gamma[\theta(t)-\theta(0)]\}\rangle\right|^{2}$ (with the fitting constant $\gamma=2$ ). We remark that $\mathcal{F}$ decays with a rate $\Lambda_{1}$ different from the Lyapunov exponent. In Fig. 1, we also plot the fidelity $\overline{F(t)}$, [see Eq. (17)], averaged over the pure Gaussian states building the initial mixture.

Note that the expected saturation values of $\bar{F}$ and $\mathcal{F}$ are $1 / N$ and $1 /(N M)$, respectively, where $N$ is the number of states in the Hilbert space and $M$ is the number of quantum cells inside the area $\Delta$. This expectation is a consequence of the randomization of phases of fidelity amplitudes and is borne out by the numerical data shown in Fig. 1.

\section{CONCLUSIONS}

In this paper we have analyzed the role of the dynamical chaos in suppressing quantum interference effects in the wave packet dynamics. We have discussed in this connection the Ramsey-type experiments with ion traps and optical lattices which allow one to directly measure the fidelity amplitudes rather than the ordinary Peres' fidelity. A measure of stability of quantum motion called allegiance has been introduced and considered in detail. This quantity directly accounts for the quantum interference as well as the dephasing effect. A nonlinear model for ion traps is proposed and investigated analytically in the coherent-state basis which represents the most adequate tool for studying the semiclassical evolution.

We have demonstrated that, starting from a fully incoherent mixture, the decay of the allegiance $\mathcal{F}$ is determined by the decay of a classical correlation function, which is totally unrelated to quantum phases. We point out that the classical autocorrelation function in Eq. (14) reproduces not only the slope but also the overall decay of the function $\mathcal{F}$. The classical dynamical variable that appears in this autocorrelation function depends on the form of the perturbation. Therefore the echo decay, even in a classically chaotic system in the semiclassical regime and with quantally strong perturbations, is to some extent perturbation dependent. The quantum dephasing described in this paper is a consequence of internal dynamical chaos and takes place in absence of any external environment. We may therefore conclude that the underlying internal dynamical chaos produces a dephasing effect similar to the decoherence due to the environment.

\section{ACKNOWLEDGMENTS}

We are grateful to Tomaž Prosen and Dima Shepelyansky for useful discussions. This work was supported in part by EU (IST-FET-EDIQIP), NSA-ARDA (ARO Contract No. DAAD19-02-1-0086) and the MIUR-PRIN 2005 "Quantum computation with trapped particle arrays, neutral and charged." V.S. acknowledges financial support from the RAS Joint scientific program "Nonlinear dynamics and solitons."
[1] F. Haake, Quantum Signatures of Chaos, 2nd. ed. (SpringerVerlag, Berlin, 2000).

[2] Quantum Chaos: Between Order and Disorder, edited by G. Casati and B. V. Chirikov (Cambridge University Press, Cambridge, England, 1995).

[3] M. C. Gutzwiller, Chaos in Classical and Quantum Mechanics
(Springer-Verlag, Berlin, 1991).

[4] A. Peres, Phys. Rev. A 30, 1610 (1984).

[5] R. A. Jalabert and H. M. Pastawski, Phys. Rev. Lett. 86, 2490 (2001).

[6] Ph. Jacquod, P. G. Silvestrov, and C. W. J. Beenakker, Phys. Rev. E 64, 055203(R) (2001). 
[7] N. R. Cerruti and S. Tomsovic, Phys. Rev. Lett. 88, 054103 (2002).

[8] T. Prosen and M. Žnidarič, J. Phys. A 35, 1455 (2002).

[9] G. Benenti and G. Casati, Phys. Rev. E 65, 066205 (2002).

[10] P. G. Silvestrov, J. Tworzydło, and C. W. J. Beenakker, Phys. Rev. E 67, 025204(R) (2003).

[11] J. Vaníček and E. J. Heller, Phys. Rev. E 68, 056208 (2003); J. Vaníček, ibid. 70, 055201(R) (2004).

[12] F. M. Cucchietti, D. A. R. Dalvit, J. P. Paz, and W. H. Zurek, Phys. Rev. Lett. 91, 210403 (2003).

[13] W. G. Wang, G. Casati, and B. Li, Phys. Rev. E 69, 025201(R) (2004).

[14] A. Iomin, Phys. Rev. E 70, 026206 (2004).

[15] B. Eckhardt, J. Phys. A 36, 371 (2003).

[16] G. Benenti, G. Casati, and G. Veble, Phys. Rev. E 67, 055202(R) (2003).

[17] S. A. Gardiner, J. I. Cirac, and P. Zoller, Phys. Rev. Lett. 79, 4790 (1997).

[18] S. Schlunk, M. B. d'Arcy, S. A. Gardiner, D. Cassettari, R. M. Godun, and G. S. Summy, Phys. Rev. Lett. 90, 054101 (2003).

[19] M. F. Andersen, A. Kaplan, and N. Davidson, Phys. Rev. Lett. 90, 023001 (2003); M. F. Andersen, A. Kaplan, T. Grünzweig, and N. Davidson, e-print quant-ph/0404118.

[20] S. Montangero, A. Romito, G. Benenti, and R. Fazio, Europhys. Lett. 71, 893 (2005).

[21] E. Schrödinger, Ann. Phys. 79, 361 (1926); 79, 489 (1926);
79, 734 (1926).

[22] P. Carruthers and M. Nieto, Rev. Mod. Phys. 40, 411 (1968).

[23] V. V. Sokolov, Teor. Mat. Fiz. 61, 128 (1984); [Theor. Math. Phys. 61, 1041 (1985)].

[24] The fidelity for a pure coherent initial state was computed in [14]. In that paper, however, a random Gaussian perturbation was considered instead of a deterministic one.

[25] M. H. Miller, J. Phys. Chem. A 105, 2942 (2001).

[26] R. J. Glauber, Phys. Rev. Lett. 10, 84 (1963).

[27] R. Z. Sagdeev, D. A. Usikov, and G. M. Zaslavsky, Nonlinear Physics (Harwood Academic, Chur, Switzerland, 1988), p. 207.

[28] F. M. Izrailev, Phys. Rep. 196, 299 (1990).

[29] F. L. Moore, J. C. Robinson, C. F. Bharucha, B. Sundaram, and M. G. Raizen, Phys. Rev. Lett. 75, 4598 (1995).

[30] H. Ammann, R. Gray, I. Shvarchuck, and N. Christensen, Phys. Rev. Lett. 80, 4111 (1998).

[31] J. Ringot, P. Szriftgiser, J. C. Garreau, and D. Delande, Phys. Rev. Lett. 85, 2741 (2000).

[32] M. B. d'Arcy, R. M. Godun, M. K. Oberthaler, D. Cassettari, and G. S. Summy, Phys. Rev. Lett. 87, 074102 (2001).

[33] M. Bienert, F. Haug, W. P. Schleich, and M. G. Raizen, Phys. Rev. Lett. 89, 050403 (2002).

[34] F. Haug, M. Bienert, W. P. Schleich, T. H. Seligman, and M. G. Raizen, Phys. Rev. A 71, 043803 (2005). 